\title{
Conventionality and Reality
}

\author{
Pieter Thyssen ${ }^{1}$
}

Received: 4 June 2019 / Accepted: 24 August 2019 / Published online: 3 September 2019 (c) The Author(s) 2019

\begin{abstract}
The debate on the conventionality of simultaneity and the debate on the dimensionality of the world have been central in the philosophy of special relativity. The link between both debates however has rarely been explored. The purpose of this paper is to gauge what implications the former debate has for the latter. I show the situation to be much more subtle than was previously argued, and explain how the ontic versus epistemic distinction in the former debate impacts the latter. Despite claims to the contrary, I conclude that special relativity leaves the debate on the dimensionality of the world underdetermined.
\end{abstract}

Keywords Special relativity $\cdot$ Conventionality of simultaneity $\cdot$ Rietdijk-Putnam argument $\cdot$ Weingard-Petkov argument $\cdot$ Presentism $\cdot$ Eternalism

\section{Introduction}

Two debates have been central in the philosophy of special relativity (SR):

1. the debate on the conventionality of simultaneity;

2. the debate on the dimensionality of the world.

The former debate was sparked by Einstein in 1905; the latter debate was initiated by Minkowski in 1908, a century ago. Einstein believed the notion of simultaneity to be conventional, and not factual; Minkowski considered reality to be fundamentally four-dimensional, and not three-dimensional.

Both debates have lingered on to this day, without definite answers. A major contribution to the second debate, in support of Minkowski's claim, came from Rietdijk [37] and Putnam [29]. Call this the RP argument. Yet another argument for the fourdimensionality of the world came from Weingard [46] and Petkov [26,27]. Call this the WP argument. While these arguments are responsible for the commonly held opinion

\footnotetext{
Pieter Thyssen

pieter.thyssen@kuleuven.be

1 Institute of Philosophy, KU Leuven, Kardinaal Mercierplein 2, 3000 Leuven, Belgium
} 
that SR necessitates a four-dimensional view of reality, neither argument is without problems, as I will show in this paper.

Most strikingly, though, the link between both debates has remained largely underexplored. To make matters even worse, whenever the link was explored, radically different conclusions were reached about the way the former debate impacts the latter. According to Weingard [46] and Petkov [26,27], for example, the conventionality thesis lends further support to Minkowski's claim. Ben-Yami [1] and Cohen [5] disagree, arguing for the opposite thesis, whereas Sklar [42] remains largely uncommitted.

The purpose of this paper then is to clarify the current situation by further exploring what implications (if any) the conventionality of simultaneity has for the debate on the dimensionality of the world.

\subsection{Outline}

Section 2 briefly reviews the debate on the conventionality of simultaneity. Section 3 provides a short introduction to the debate on the dimensionality of the world. Section 4 outlines the RP argument, and Sect. 5 raises a number of objections against it. Most importantly among these is the conventionality objection according to which the conventionality thesis undermines the RP argument. Section 6 shows the situation to be much more subtle than that, and explains how the ontic-versus-epistemic distinction in the former debate impacts the latter. Section 7 summarises the WP argument, and Sect. 8 briefly mentions the transitivity objection. Section 9 concludes this paper with some final thoughts on the soundness of the RP and WP argument.

\section{The Conventionality of Simultaneity}

The claim that distant simultaneity is a conventional notion (as opposed to a factual one) originated in the writings of Poincaré and Einstein, and was further developed by Reichenbach in the 1920 s and by Grünbaum in the 1950s [19]. ${ }^{1}$ The conventionality thesis can be summarised as follows. Consider two distant events, one at location $A$ in space, the other at location $B$. To say that both events are simultaneous is to say that they occur at the same time. That is, if an $A$ - and a $B$-clock were placed at the locations $A$ and $B$ respectively, both clocks should indicate the same time. This of course presumes that the clocks have been previously synchronised.

\subsection{Clock-Synchronisation}

In his 1905 paper, Einstein [12] proposed the following clock-synchronisation procedure (Fig. 1). At time $t_{A}$, a light signal is emitted from point $A$ towards point $B$ (event $e_{A}$ ). At time $t_{B}$, the signal is reflected back from $B$ to $A$ (event $e_{B}$ ) and returns at $A$ at time $t_{A^{\prime}}$ (event $e_{A^{\prime}}$ ). Notice that the times $t_{A}$ and $t_{A^{\prime}}$ are measured by the $A$-clock,

\footnotetext{
1 The conventionality of simultaneity should not be confused with the relativity of simultaneity. Whereas the latter refers to the relativity of intersystemic simultaneity, the former refers to the relativity of intrasystemic simultaneity.
} 
Fig. 1 Standard synchrony as defined by Einstein in 1905

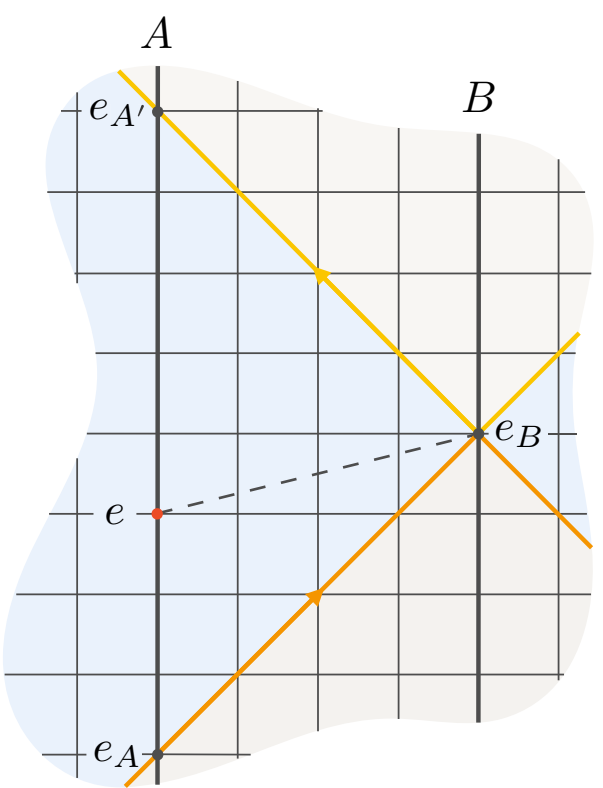

whereas the time $t_{B}$ is measured by the $B$-clock. If the speed of light is the same in the $A B$ and $B A$ directions, it follows that the two clocks are synchronous when

$$
t_{B}=t_{A}+\frac{1}{2}\left(t_{A^{\prime}}-t_{A}\right)
$$

\subsection{The Conventionality Thesis}

Einstein's procedure however relies on an important assumption: the isotropy of the speed of light. In order to verify the truth of this assertion, the one-way velocity of light would have to be measured. But this requires the use of spatially separated clocks that are already synchronised. As Einstein [11, p. 27] observed: "It would thus appear as though we were moving here in a logical circle." Reichenbach called this the 'velocity-simultaneity circle argument'. Einstein avoided the circularity by assuming the isotropy of the velocity of light without further (experimental) proof. ${ }^{2}$ Einstein's definition of distant simultaneity is thus only a convention. Other definitions are possible according to which

$$
t_{B}=t_{A}+\varepsilon\left(t_{A^{\prime}}-t_{A}\right), \quad 0<\varepsilon<1,
$$

with $\varepsilon$ the Reichenbach synchronisation parameter. The choice $\varepsilon=\frac{1}{2}$ is called standard synchrony and leads to Einstein's definition of simultaneity. But according to

\footnotetext{
2 Einstein was probably aware of the conventional character of his synchronisation procedure. He was careful, after all, to use the words "by definition" when establishing the isotropy of the speed of light, and titled the first section of his 1905 paper " $\$ 1$. Definition of Simultaneity" (see [12, p. 142]).
} 

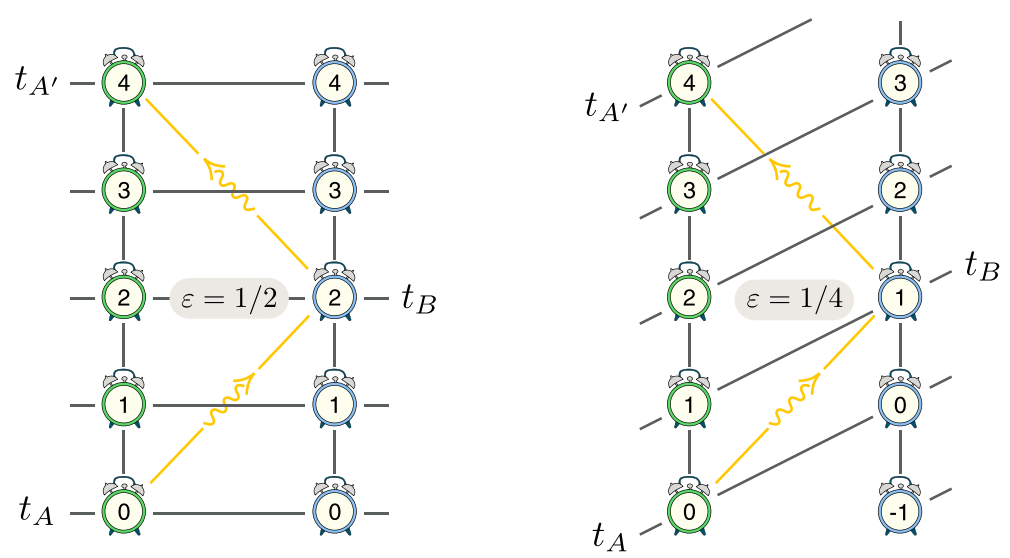

Fig. 2 Standard $\varepsilon=\frac{1}{2}$ versus non-standard $\varepsilon=\frac{1}{4}$ synchrony. Figure adapted from Norton [23]

Reichenbach, the choice of $\varepsilon$ is completely arbitrary (see Fig. 2). This, in short, is the conventionality thesis of simultaneity.

\subsection{The Causal Theory of Time}

Reichenbach arrived at the conventionality thesis via a different route. ${ }^{3}$ According to his causal theory of time, all temporal relations are reducible to causal relations. An event $e_{1}$ is earlier than an event $e_{2}$ if and only if $e_{1}$ can causally affect $e_{2}$. Since $e_{A}$, $e_{B}$, and $e_{A^{\prime}}$ in Fig. 1 are connected via a light signal, $e_{A}$ can affect $e_{B}$ and $e_{B}$ can affect $e_{A^{\prime}}$. It follows that $t_{A}<t_{B}<t_{A^{\prime}}$. But for any event $e$ in the open interval between $e_{A}$ and $e_{A^{\prime}}, e$ can only affect $e_{B}$, or vice versa, if a causal signal were to travel between them at superluminal speeds, which is forbidden according to SR. It is this causal non-connectibility of $e$ and $e_{B}$ that leaves their temporal order indeterminate according to Reichenbach. The event $e$ is neither past, present, nor future with respect to $e_{B}$.

In summary, the temporal order for any two spacelike separated events is indeterminate. It is only when a definition of distant simultaneity is introduced by hand (via a conventional choice of $\varepsilon$ ) that a temporal order between spacelike separated events can be established. But this order merely reflects our choice of $\varepsilon$, rather than being an objective matter of fact.

\subsection{Malament}

The conventionality thesis, it must be said, is not universally accepted. The most influential objection was probably voiced by Malament [20]. According to Norton [22, p. 194], Malament's publication represented "one of the most dramatic reversals in the philosophy of space and time." It is not my aim in this paper to take a position with regard to the conventionality debate; I merely want to point out what impact the

\footnotetext{
3 See Reichenbach [31-33] (translated in [34-36] respectively).
} 

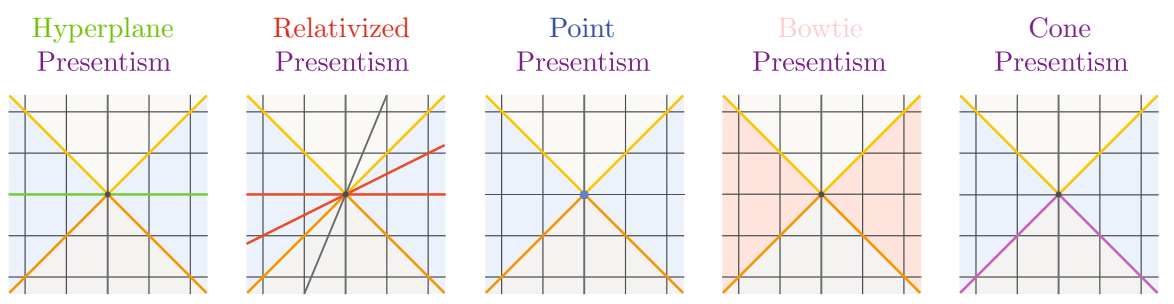

Fig. 3 Different flavours of presentism

conventionality thesis has on the debate about the dimensionality of the world if it were true.

\section{What is Real?}

One of the central questions in the philosophy of SR is the reality question: is only the present real (presentism), or are the past and future equally real (eternalism)? There are of course other metaphysical positions, such as the view that the past and present are real (possibilism). Also, presentism is an umbrella term, covering a wide range of different views. Depending on which spatiotemporal shape the present takes on, for instance, different flavours of presentism are obtained (Fig. 3). Some of these flavours will be discussed further on. But for the moment, I want to keep the discussion focussed, and will take the present to be a three-dimensional Cauchy hyperplane, spanning the entire spatial extent of the world. Call this the hyperplane present. With that in place, let me briefly unpack the standard presentist and eternalist view.

\subsection{Presentism}

On the presentist view, the present is singled out as a uniquely special moment we call NOW. Only those events that constitute the present moment are real. Past events are no longer real and future events are not yet real. According to hyperplane presentism, the world, as a consequence, is three-dimensional. ${ }^{4}$

Notice also that presentism is a realist thesis [38]: there is an objective, universal fact of the matter as to which events constitute the present moment, whether or not we have epistemic access to it. That is, the presentist thesis makes an ontological claim about the nature of time, not an epistemological one.

In presentism, time is usually assumed to pass: present events disappear into the past as future events come into existence, leading to a succession of presents or a moving NOw. This dynamic aspect of time is referred to as the passage of time or temporal becoming. Change and temporal becoming are thus taken to be fundamental aspects of reality. The passage of time, however, is not logically entailed by the belief

\footnotetext{
4 Not all presentists would agree on this: according to the point presentist, the world is zero-dimensional; for the bowtie and cone presentists, the world is four-dimensional.
} 
that only the present exists (see [21]). In any case, our focus here is on the reality of events and the dimensionality of the world, not on becoming.

\subsection{Eternalism}

On the eternalist view, all past, present, and future events are equally real and determinate. No special status is accorded to the present moment. The world, as a consequence, is four-dimensional. The eternalist account of time finds a natural representation in the so-called block universe, where all events coexist on an equal footing. From a God's eye point of view — or what Price [28] calls the view from nowhen-every moment of the universe's history is set out, and time no longer flows. Reality, in the words of Black [2, p. 181], is "a timeless web of 'world-lines' in a four-dimensional space."

\subsection{What is Real?}

The difference between presentism and eternalism is thus cashed out in terms of which events are real. For the presentist, the events simultaneous with the here-and-now are real. For the eternalist, all events are real, whether or not they are simultaneous with the here-and-now.

But what exactly does it mean to say that a particular event is real? This question has remained largely untouched in the philosophical literature. Two exceptions are Callender [4] and Peterson and Silberstein [25]. Callender asks us to consider a fourdimensional manifold of events, where each event carries a lightbulb that can be ON or OFF. When a lightbulb is ON, the corresponding event is real; when the lightbulb is OFF, the event is not real. Presentism, on this view, holds that only present lights are ON, whereas eternalism maintains that all lights are ON (Fig. 4). ${ }^{5}$

\subsection{Reality Values and Relations}

Instead of associating a lightbulb with each event, Peterson and Silberstein [25] introduce a reality field $R$ which denotes the ontological status of each event by assigning it a dimensionless reality value or $R$-value:

$$
\begin{aligned}
R: \mathcal{M} & \longrightarrow[0,1] \\
a \in \mathcal{M} & \longmapsto R(a)
\end{aligned}
$$

Since the reality field is a scalar field, all observers agree on the value of the reality field at a particular point of spacetime. Every event, in other words, has a unique $R$ value, with $R=1$ denoting a real event, and $R=0$ an unreal event (Fig. 5). This is called the uniqueness criterion.

Peterson and Silberstein next introduce a binary reality relation $R$ which holds between any two events having the same $R$-value. For instance, if $a, b \in \mathcal{M}$ share the

\footnotetext{
5 Possiblism is an intermediate position between presentism and eternalism, arguing that only past and present lights are $\mathrm{ON}$.
} 

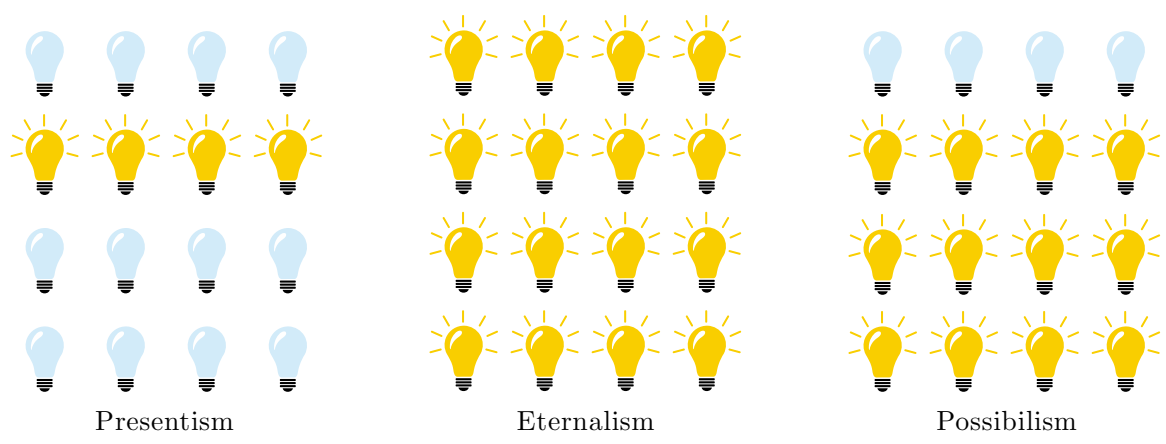

Fig. 4 Which lightbulbs are ON?

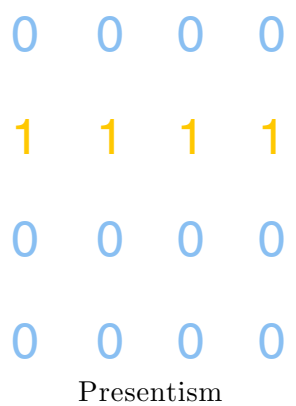

Fig. 5 Reality values

same $R$-value, then they are said to be equally real. This is written as $a R b$ (read: 'event $a$ and event $b$ are equally real' or 'event $a$ is real for event $b$ '). Due to the uniqueness criterion, the relation $R$ is:

1. Reflexive: $a R a$ is true (since $a$ has a unique $R$-value);

2. Symmetric: if $a R b$ is true, then $b R a$ is true (since $a$ and $b$ share the same $R$-value);

3. Transitive: if $a R b$ is true, and $b R c$ is true, then $a R c$ is true (since $a$ and $c$ share the same $R$-value).

This turns $R$ into an equivalence relation. As a consequence, $R$ provides a partition of the underlying set $\mathcal{M}$ into two disjoint equivalence classes: the class of real events and the class of unreal events.

\subsection{The Presentist Credo}

With this in place, we can rewrite the presentist credo that all (and only) present events are real more explicitly. Let $\mathcal{M}$ be the set of all spacetime events $a, b, \ldots$, and $S$ the relation of simultaneity among the elements of $\mathcal{M}$. Then $a S b$ is shorthand for 'event $a$ is simultaneous with event $b$ '. If $b$ represents the here-and-now, $b$ is real. That is, $R(b)=1$. The present for $b$ consists of all events simultaneous with $b$. Hence, if $a S b$ holds true, then $a$ is present for $b$. Following the presentist credo, $a$ is therefore real for $b$ : 


$$
a S b \Longrightarrow a R b,
$$

with $R(a)=R(b)=1$. Call this hyperplane presentism.

\section{The Rietdijk-Putnam Argument}

Presentism is often said to be closest to our common-sense beliefs and intuitions about time. Putnam [29, p. 240] thus calls it the view of "the man on the street." But with the advent of SR, the presentist position has come under increasing pressure. The relativity of simultaneity, in particular, challenges our presentist intuitions and seems to imply an eternalist picture of time instead. According to Savitt [40], the eternalist account of time is now the most popular among philosophers.

\subsection{The Rietdijk-Putnam Argument}

One of the best known arguments from SR in favour of eternalism and the fourdimensionality of the world is the so-called Rietdijk-Putnam (RP) argument [29,37]. ${ }^{6}$ The RP argument is a reductio ad absurdum (but see Stein [44, p. 17]). Rietdijk and Putnam start from the presentist doctrine according to which all (and only) present events are real and determinate (future and past events being indeterminate) and proceed to show the untenability of this position in light of SR.

The argument relies on the well-known relativity of simultaneity: for any event that is future with respect to one observer, there always is a second observer (simultaneous with the first) for whom that event is present and hence (following the presentist credo) real. But surely - the argument continues - if an event is real for one observer, it has to be real for all observers. Thus, Putnam [29, p. 242] concludes: "future things (or events) are already real." The same can of course be said for past events, implying that future and past events are real after all. This refutes presentism, and confirms eternalism.

Let us go through the argument in a bit more detail. Consider the set $\mathcal{M}$ of spacetime events $a, b, \ldots$, and let $S$ and $R$ be the relations of simultaneity and reality as defined above. Now, let $a$ and $b$ be two events on the worldline of an inertial observer $\mathcal{O}_{1}$ such that $a$ chronologically precedes $b$ (Fig. 6). Consider a second observer $\mathcal{O}_{2}$ with an event $c$ on her worldline that is spacelike separated from both $a$ and $b$, such that:

(i) At $a, c$ is present relative to $\mathcal{O}_{1}$ and is therefore real for $\mathcal{O}_{1}$;

(ii) At $c, b$ is present relative to $\mathcal{O}_{2}$ and is therefore real for $\mathcal{O}_{2}$.

Due to the transitivity of the relation 'is real for', it follows from (i) and (ii) that:

(iii) At $a, b$ is real for $\mathcal{O}_{1}$.

But $b$ is in the chronological future of $a$. Hence, on a presentist reading:

(iv) At $a, b$ is not real for $\mathcal{O}_{1}$.

\footnotetext{
${ }^{6}$ A well-known variation on the Rietdijk-Putnam theme is the Andromeda paradox, which was put forward by Penrose [24, pp. 392-393].
} 


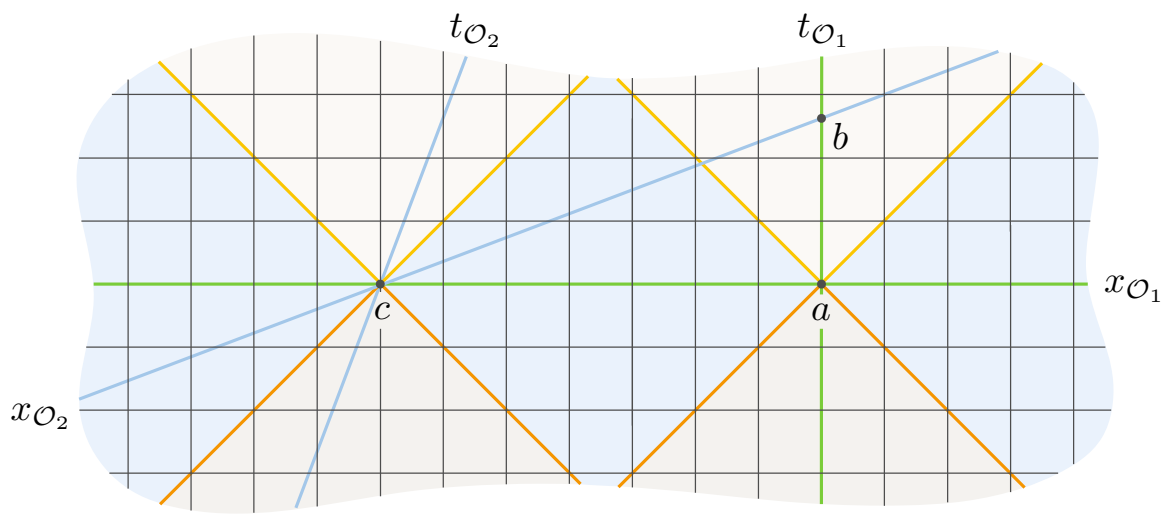

Fig. 6 The Rietdijk-Putnam argument

A contradiction arises between (iii) and (iv), thereby refuting (hyperplane) presentism and establishing the eternalist worldview instead. Rewriting the RP argument in shorthand notation yields:

(i) $c S a \Longrightarrow c R a$;

(ii) $b S c \Longrightarrow b R c$;

(iii) $b R c \wedge c R a \Longrightarrow b R a$;

(iv) $\neg b S a \Longrightarrow \neg b R a$;

(v) $b R a \wedge \neg b R a$.

\section{Against Rietdijk-Putnam}

Although the RP argument claims to have settled the debate on the side of eternalism, a number of important objections have been raised against it, exposing different fallacies in the RP argument. I mention two objections, and will concentrate on the second.

\subsection{The Transitivity Objection}

The most common objection focusses on the transitivity of the relation 'is real for'. For-the objection runs-the present in SR is a relative (frame-dependent) notion. What is present for $\mathcal{O}_{1}$ need not be present for $\mathcal{O}_{2}$. And since the reality of events is tied up with their being present, reality itself is bound to be relativized. What is real for $\mathcal{O}_{1}$ need not be real for $\mathcal{O}_{2}$.

The non-transitivity of $R$ follows directly from the non-transitivity of $S$. Just as $b S c$ and $c S a$ in Fig. 2 does not imply that $b S a$, so $b R c$ and $c R a$ does not imply that $b R a$. To see this, recall that the relation of simultaneity in SR is a ternary (threeplace) relation among two events and a given reference frame. Two events are only simultaneous with one another relative to some observer. When this is taken into

\footnotetext{
7 See, for instance, Sklar [41], Godfrey-Smith [14], Sklar [42] (republished in Sklar [43]), Hinchliff [17,18], and Dieks [9]. 
account, the non-transitivity of $S$ across observers is immediately obvious:

$$
b S_{\mathcal{O}_{2}} c \wedge c S_{\mathcal{O}_{1}} a \Longrightarrow b S_{\mathcal{O}_{1}} a \text {. }
$$

The flaw in the RP argument-so the objection goes-is that $R$ is taken to be a binary (two-place) relation among events, and not a ternary one such as $S$, in which case:

$$
b R_{\mathcal{O}_{2}} c \wedge c R_{\mathcal{O}_{1}} a \Rightarrow b R_{\mathcal{O}_{1}} a .
$$

By making $R$ observer-dependent, there no longer is one reality, but a plurality of (observer-dependent) realities [3]. Such relativisation of existence gives rise to an ontological pluralism, as exemplified in relativized presentism (Fig. 3).

\subsection{The Conventionality Objection}

A second objection, based on the conventionality of simultaneity, recently appeared in a paper by Ben-Yami [1]. According to the conventionality thesis, the temporal order for spacelike separated events is indeterminate (see $\$ 2$ ). Hence, since $c$ is spacelike separated from $a$ in Fig. 6, it cannot be maintained that $c$ is present relative to $\mathcal{O}_{1}$ at $a$. Similarly, since $b$ is spacelike separated from $c$, it cannot be maintained that $b$ is present relative to $\mathcal{O}_{2}$ at $c$.

\subsection{Premises and Conclusion}

Notice that the RP argument falls apart under both objections, but for different reasons [1]. According to the transitivity objection, the conclusion (iii) does not follow from the premises (i) and (ii). According to the conventionality objection, the argument does not even get off the ground since both premises (i) and (ii) are considered false, rendering the argument unsound. Whereas the first objection questions the validity of the RP argument, the latter objection questions its soundness.

\subsection{Weingard and Sklar}

The conventionality objection is certainly not new, despite Ben-Yami's claim to the contrary. Weingard [46] and Sklar [42] were among the first to apply the conventionality thesis to the RP argument. More recently, Dieks [8] and Cohen [5] endorsed the same viewpoint. Here is Sklar [42, pp. 135-36]:

If we now associate the real (for an observer) with the simultaneous for him, we must, accepting the conventionality of simultaneity, accept as well a conventionalist theory of 'reality for'. It is then merely a matter of arbitrary stipulation that one distant event rather than another is taken as real for an observer. Now there is nothing inconsistent or otherwise formally objectionable about such a relativized notion of 'reality for', but it does seem to take the metaphysical heart 
out of the old claim that the present had genuine reality and the past and future lacked it. For what counts as the present is only a matter of arbitrary choice, and so then is what is taken as real.

\section{Ontic or Epistemic?}

In deciding whether the conventionality objection referred to above has any strength, one first has to decide whether the conventionality of simultaneity is an issue of ontology or epistemology.

\subsection{Ontic or Epistemic?}

On an ontic reading of the conventionality thesis, the relation of distant simultaneity is conventional, as opposed to factual, because this relation does not exist in the objective world. "[I]t is because no relations of absolute simultaneity exist to be measured that measurement cannot disclose them", argues Grünbaum [15, p. 456].

On an epistemic reading of the conventionality thesis, on the other hand, the relation of distant simultaneity is conventional because it is unverifiable. Even if the relation of distant simultaneity really exists, we nevertheless fail to have epistemic access to it, and are thus forced to treat this notion in a conventional manner.

\subsection{Agnostic or Epistemicist?}

With respect to the epistemic reading of the conventionality thesis, it is worth distinguishing two further positions. The agnostic is non-committal about the possible existence of distant simultaneity. The $\varepsilon$-epistemicist, on the other hand, is convinced that there is "a fact of the matter as to which distant events are 'really' simultaneous with a given event", even though we cannot measure it empirically. That is, the Reichenbach $\varepsilon$-parameter has a determinate value, but due to the velocity-simultaneity circle argument (referred to above, see $\S 2$ ), there is no way for us to determine its value. ${ }^{8} \mathrm{I}$ call this position $\varepsilon$-epistemicism, borrowing the term from debates on vagueness. ${ }^{9}$

\subsection{Ontic Impact}

On an ontic reading of the conventionality thesis, the conventionality objection referred to above certainly applies. After all, if distant simultaneity does not belong to the ontological furniture of the world, then clearly premises (i) and (ii) are without substance.

\footnotetext{
8 This is similar to the hidden variables in certain interpretations of quantum mechanics, such as the particle positions in Bohmian mechanics. Even though each particle always has a definite position, thereby tracing out a classical (or semi-classical) trajectory over time, we do not have epistemic access to these positions.

9 Epistemicism is a philosophical position according to which propositions involving vague predicates (such as 'is thin' or 'is a heap of sand') have definite truth values, even though it is impossible in principle to know what they are.
} 


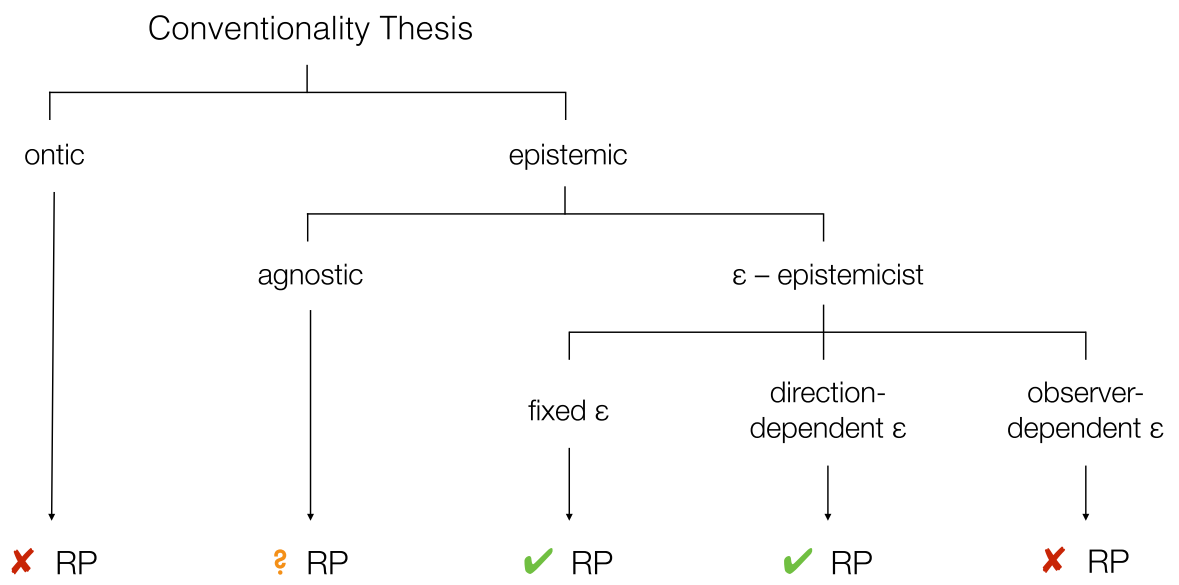

Fig. 7 The impact of the conventionality thesis on the RP argument

Not surprisingly, Weingard, Sklar, Dieks, Ben-Yami, and Cohen all subscribe to this ontological interpretation when raising their objection.

Sklar [42, p. 135], for instance, takes the simultaneity of distant events to be "irrealist." We are of course free to introduce such a notion by choosing a particular value for $\varepsilon$. But, argues Sklar, if every choice of $\varepsilon$ "can explain equally well all the hard data of experience, why should we take the accounts as differing at all in the real features they attribute to the world?" There is, in other words, "no fact of the matter at all about which distant events are 'really' simultaneous with a given event". Ben-Yami [1, p. 278] agrees that the definitions of distant simultaneity "do not express any objective temporal order between [spacelike separated] events."

But "if simultaneity is purely conventional and lacks metaphysical significance," Dieks [8, pp. 618-619] continues, "there is obviously no reason to suppose that simultaneous events share a special "reality-property", so that the Rietdijk/Putnam argument seems to become a non-starter." Cohen [5, p. 46], finally, concurs that "since simultaneity between spatially separated events is merely conventional and not an objective constituent of reality", the premises (i) and (ii) above are "devoid of physical import."

\subsection{Point Presentism}

Granting that the ontic interpretation of the conventionality thesis undermines the $\mathrm{RP}$ argument, where does it leave us with regard to the debate on presentism and eternalism? If there is no such thing as distant simultaneity of events, it would seem that the present gets reduced to the here-and-now of each observer. And if we accept the presentist credo that all that exists, exists presently, then reality itself would get reduced to a single point (Fig. 3). This was called point presentism by Harrington [16]. The problem, according to Stein [44, p. 18], is that it leads to "a peculiarly extreme (but pluralistic!) form of solipsism." 
Fig. 8 The Rietdijk-Putnam argument with $\varepsilon=\frac{1}{4}$

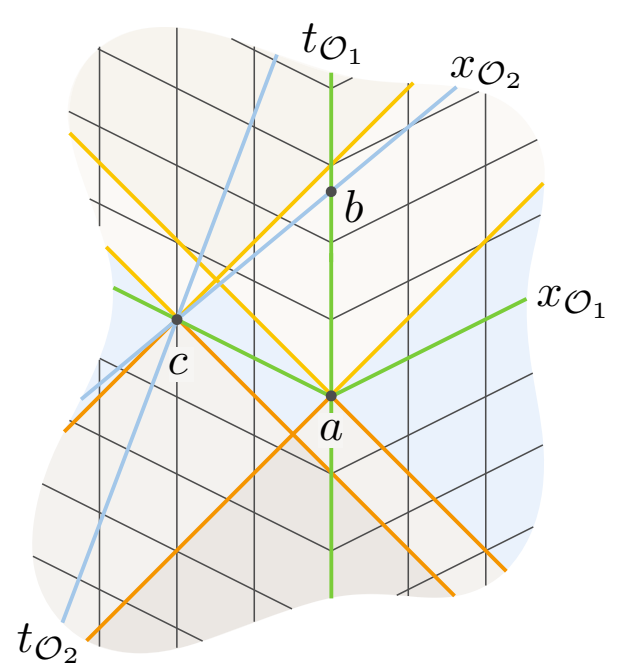

Not everyone has reached this conclusion though. Weingard [46], for instance, while agreeing that the conventionality thesis undermines the RP argument, offers a new argument, based on the conventionality thesis, in support of eternalism (see $§ 7) .{ }^{10}$

\subsection{Epistemic Impact}

Let us first turn to the epistemic interpretation of the conventionality thesis and its consequences for the RP argument. Here the situation becomes more subtle (Fig. 7). Agnostics cannot judge the soundness of the RP argument since they are undecided whether distant simultaneity really exists.

The $\varepsilon$-epistemicists, on the other hand, can go both ways. If they assume that $\varepsilon$ has a fixed value, different from $\frac{1}{2}$, then the conventionality objection fails, and the RP argument nevertheless goes through. To see that, compare Figs. 6 and 8. Rietdijk and Putnam both assume standard synchrony with $\varepsilon=\frac{1}{2}$, leading to the familiar hyperplanes of simultaneity which are orthogonal to the worldlines of the observers (Fig. 6). But suppose now that $\varepsilon$ had a different value in reality, say $\varepsilon=\frac{1}{4}$. In that case, spacetime would be foliated into one-sheeted hypercones of simultaneity (Fig. 8). ${ }^{11}$ Yet, despite such a different foliation, the relativity of simultaneity still holds true, and the RP argument goes through unaffected.

One problem with the hypercones is that the notion of intrasystemic simultaneity is no longer symmetric and transitive, and thus no longer an equivalence relation. Although $c$ is simultaneous with $a$ in Fig. $8(c S a)$, for example, $a$ is not simultaneous with $c(\neg a S c)$. It is customary therefore to make $\varepsilon$ direction-dependent (with a choice of $\varepsilon=\frac{1}{4}$ to the right implying $1-\varepsilon=\frac{3}{4}$ to the left, as explained by Dieks [9]). This

\footnotetext{
10 Sklar [42] also voices a number of ways to deal with the threat of conventionality.

11 Only for standard synchrony with $\varepsilon=\frac{1}{2}$ do the hypercones degenerate into the familiar horizontal hyperplanes of simultaneity (see $[30,45])$.
} 


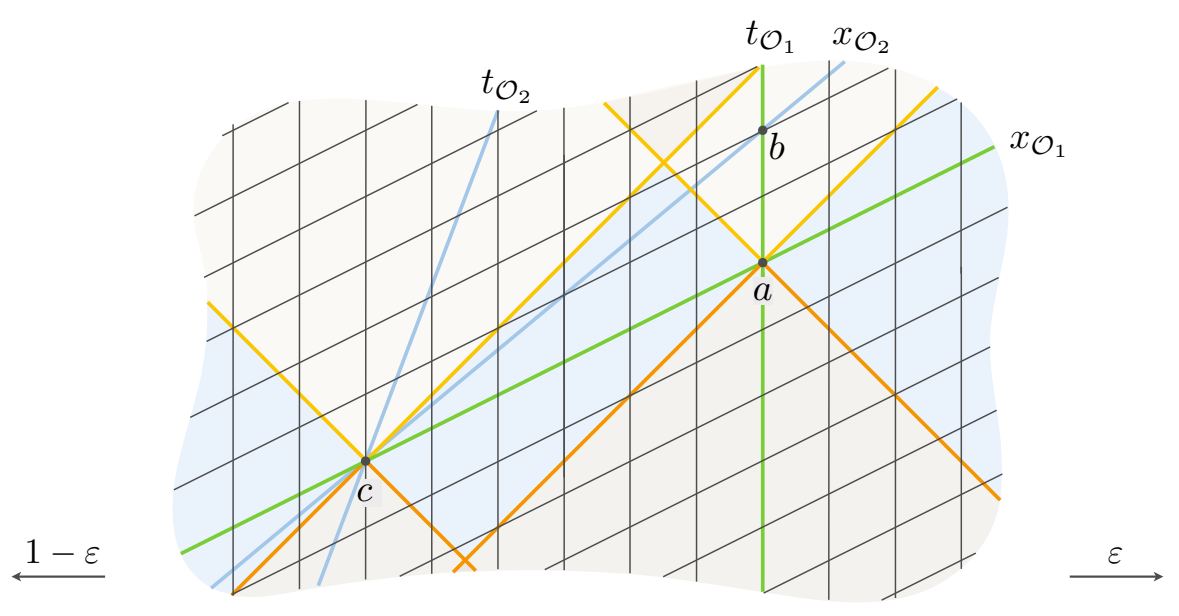

Fig. 9 The Rietdijk-Putnam argument with direction-dependent $\varepsilon$

leads to a foliation of Minkowski spacetime into hyperplanes, rather than hypercones, which are not orthogonal to the time axis. Even so, the relativity of simultaneity still holds true, and the RP argument applies (Fig. 9).

However, since the choice of $\varepsilon$ is conventional, nothing prevents the epistemicist from making $\varepsilon$ observer-dependent as well. That way, a notion of absolute simultaneity can be reintroduced, in which case the RP argument obviously fails (Fig. 10). Neo-Lorentzian interpretations of SR, in particular, subscribe to this position (see for instance Craig [6], Craig and Smith [7]). The threat of nonlocality has also led some Bohmians to introduce a preferred foliation of spacetime [10].

\section{The Weingard-Petkov Argument}

Another argument from SR for the four-dimensionality of the world is the WeingardPetkov (WP) argument, which was first proposed by Weingard [46] and has since been advocated by Petkov [26,27]. Whereas the RP argument relies on the relativity of simultaneity, the WP argument relies on the conventionality of simultaneity.

Weingard [46] takes issue with the RP argument for two reasons. First, RP use the relation of distant simultaneity $S$ to partition Minkowski spacetime into past, present and future. But the relation of distant simultaneity is frame-dependent, and hence not relativistically invariant. Two observers in relative motion will carve Minkowski spacetime differently, and so won't agree on what is past, present or future. Hence, according to Weingard, our ontology should never be based on frame-dependent concepts, but always on invariant ones.

Secondly, Weingard was the first to raise the conventionality objection, as described in $\$ 5$. Even for one and the same observer, what is past, present and future in the absolute elsewhere is conventional, and hence devoid of ontological significance. Despite this fact, Weingard also uses the conventionality of simultaneity constructively to give a modified argument in support of eternalism. 


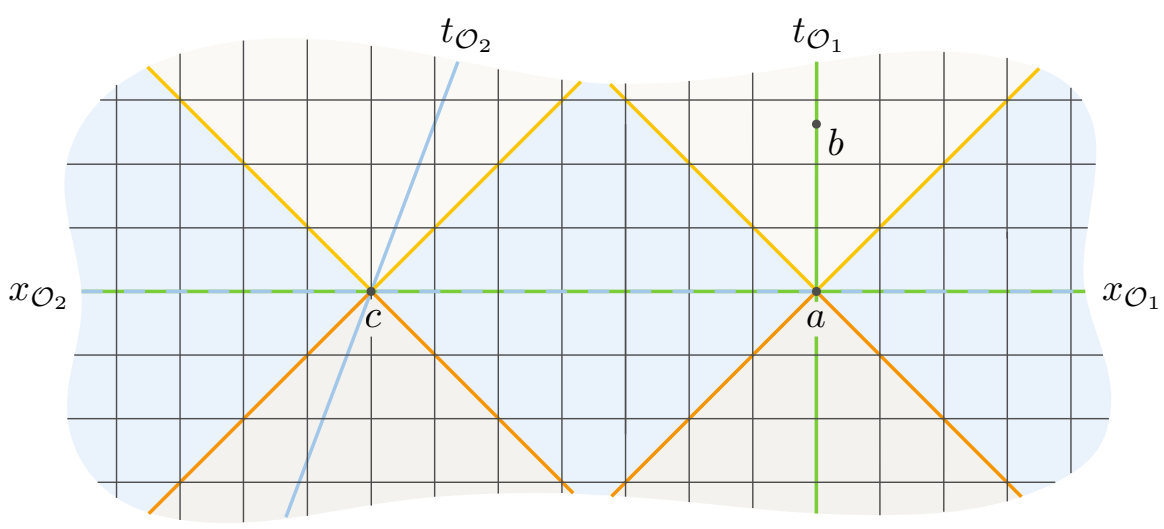

Fig. 10 The Rietdijk-Putnam argument with observer-dependent $\varepsilon$

\subsection{Topological Simultaneity}

Consider the set $\mathcal{M}$ of spacetime events $a, b, \ldots$, and let $b$ represent the here-and-now. By carefully choosing $\varepsilon$, any event in the absolute elsewhere of $b$ can be considered simultaneous with $b$, and hence present. The present for $b$, in other words, is just the absolute elsewhere of $b$-a spatially extended bowtie-shaped region (Fig. 3). It contains all events that are causally non-connectible to $b$, and hence (in the words of Reichenbach and Grünbaum) topologically simultaneous with $b$.

Contrary to the (standard $\varepsilon=\frac{1}{2}$ ) hyperplane present for $b$, the bowtie present for $b$ is relativistically invariant. It neatly partitions Minkowski spacetime into an absolute present $(b+$ elsewhere of $b)$, absolute future (upper lightcone of $b$ ) and absolute past (lower lightcone of $b)^{12}$

\subsection{Bowtie Presentism}

Let $A$ be the relation among the elements of $\mathcal{M}$ where $A$ stands for "is in the absolute elsewhere of'. Then $a A b$ is shorthand for 'event $a$ is in the absolute elsewhere of event $b$ '. Since $b$ represents the here-and-now, $b$ is real. The present for $b$ consists of all events topologically simultaneous with $b$. Hence, if $a A b$ holds true, then $a$ is present for $b$. Following the presentist credo that all (and only) present events are real, $a$ must be real for $b$ :

$$
a A b \Longrightarrow a R b \text {. }
$$

This position was dubbed bowtie presentism by Gilmore et al. [13].

\footnotetext{
12 Savitt [39] rejects the bowtie present because it fails to be achronal. According to him, no events in the present of $b$ should be in each other's absolute future or absolute past. To see why, imagine that your entire worldline from birth to death was contained in the absolute elsewhere of $b$. Then according to $b$, your entire life is present, which sounds absurd.
} 
Fig. 11 The Weingard-Petkov argument

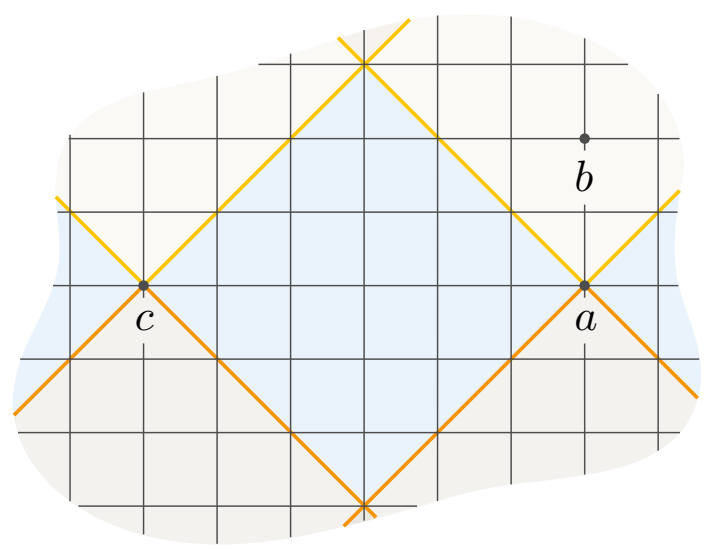

\subsection{The Weingard-Petkov Argument}

The WP argument, in essence, is just the RP argument, but using (7) instead of (4) to gauge what is real (Fig. 11):

(i) $c \mathrm{Aa} \Longrightarrow c \mathrm{Ra}$;

(ii) $b A c \Longrightarrow b R c$;

(iii) $b R c \wedge c R a \Longrightarrow b R a$;

(iv) $\neg b A a \Longrightarrow \neg b R a$;

(v) $b R a \wedge \neg b R a$.

Once again, a contradiction arises in $(\mathrm{C})$, thereby refuting bowtie presentism and establishing eternalism.

\section{Against Weingard-Petkov}

\subsection{The Transitivity Objection}

Although the conventionality objection does not apply to the WP argument, the transitivity objection still applies. For even the bowtie present is a relative notion. The bowtie present for $a$ in Fig. 11, after all, is different from the bowtie present for $c$. Hence, if the reality of events is tied up with their being present, then what is real for $a$ need not be real for $c$.

Here again, the non-transitivity of $R$ follows directly from the non-transitivity of $A$. That is, starting from

$$
b A c \wedge c A a \Longrightarrow b A a,
$$

and applying (7), one obtains:

$$
b R c \wedge c R a \Longrightarrow b R a,
$$

in contradiction with premise (iii) in the above WP argument. 


\subsection{Reality Relations}

If the transitivity objection holds true, then why do Peterson and Silberstein [25] uphold the transitiveness of the reality relation $R$, as we showed in $\S 3$ ? The reason, quite simply, is that Peterson and Silberstein force $R$ to be transitive by requiring every spacetime event to have a unique $R$-value. ${ }^{13}$ This uniqueness criterion "seems intuitive" enough, write Peterson and Silberstein [25, p. 212], "since an event with an $R$-value of both 1 and 0 , on our scheme, would be both real and unreal, which would be a contradiction."

But intuitions are not always the most reliable guide to ontology. Perhaps an event can have an $R$-value of both 1 and 0 , depending on which point of view one considers. To a bowtie presentist, for instance, the event $b$ in Fig. 11 is real for $c$, but unreal for $a$. Although a rejection of the uniqueness criterion thus leads to a relativization of existence, "there doesn't seem to be anything very objectionable a priori about this", dixit Sklar [43, p. 296]. The question whether the reality relation $R$ is transitive or not thus remains very much open.

\section{Conclusions}

The purpose of this paper was to explore the link between two major debates in the philosophy of SR: the debate on the conventionality of simultaneity and the debate on the dimensionality of the world. The focus here was on the RP and WP arguments. Both arguments claim that SR necessitates an eternalist, four-dimensional view of reality.

According to Weingard, Sklar, Ben-Yami and others, the conventionality of simultaneity undermines the RP argument. I have shown the situation to be more subtle than that and have argued that the way in which the conventionality thesis impacts the RP argument depends on whether it is an ontological or epistemological thesis. If it is an ontological thesis, the RP argument cannot be saved. But on certain epistemicist positions regarding distant simultaneity, the RP argument is unaffected by the conventionality objection (Fig. 7).

Even then, both the RP and WP argument remain subject to other objections, the transitivity objection being just one example. Here, the soundness of both arguments hinges on our interpretation of reality, and in particular on the alleged transitivity of the reality relation $R$. Since this relation does not belong to the formalism of SR, SR alone cannot answer the reality question. Indeed, despite claims to the contrary, SR leaves the debate on the dimensionality of the world underdetermined.

What is needed in order to answer the reality question are additional metaphysical assumptions and presuppositions, which fall outside the scope of SR. This conclusion beautifully resonates with Sklar [41, pp. 272-275]:

[S]pecial relativity throws novel light on the philosophical questions, but it is unable by itself to resolve fully the long-standing philosophical issues. [...] The

\footnotetext{
13 After all, if $b R c$ means that $b$ and $c$ have the same $R$-value, and $c R a$ means that $c$ and $a$ have the same $R$-value, and $b, c$ and $a$ all have a unique $R$-value, then clearly $b$ and $a$ must have the same $R$-value as well.
} 
science can change the philosophy and put the dispute in a new perspective, but

it cannot resolve the dispute in any ultimate sense.

Acknowledgements I owe a great debt to Dennis Dieks for his time and careful remarks, which greatly improved this paper. I also wish to thank the participants of the Fifth International Conference on the Nature and Ontology of Spacetime (Albena, Bulgaria, May 14-17, 2018) for stimulating discussions. I am also indebted to Sylvia Wenmackers and Gerard Bodifee for useful comments on earlier versions of this paper. This work was supported by the Research Foundation Flanders (Fonds Wetenschappelijk Onderzoek, FWO), grant number G0B8616N “Time, Causality, and Probability in Quantum Mechanics: Assessing Retrocausal Explanations in Light of Recent Experiments".

Open Access This article is distributed under the terms of the Creative Commons Attribution 4.0 International License (http://creativecommons.org/licenses/by/4.0/), which permits unrestricted use, distribution, and reproduction in any medium, provided you give appropriate credit to the original author(s) and the source, provide a link to the Creative Commons license, and indicate if changes were made.

\section{References}

1. Ben-Yami, H.: Causal order, temporal order, and becoming in special relativity. Topoi 34, 277-281 (2015)

2. Black, M.: Review of G. J. Whitrow's the natural philosophy of time. Sci. Am., CCVI(April):181-182 (1962)

3. Bouton, C.: Is the future already present? the special theory of relativity and the block universe view. In: Bouton, C., Huneman, P. (eds.) Time of Nature and the Nature of Time: Philosophical Perspectives of Time in Natural Sciences. Boston Studies in the Philosophy and History of Science, Book Section 6, pp. 89-122. Springer, Switzerland (2017)

4. Callender, C.: Shedding light on time. Philos. Sci. 67, S587-S599 (2000)

5. Cohen, Y.: Why presentism cannot be refuted by special relativity. In: Dolev, Y., Roubach, M., (eds), Cosmological and Psychological Time, pp 41-51. Springer, Cham (2016). https://doi.org/10.1007/ 978-3-319-22590-6_3

6. Craig, W.L.: Time and the Metaphysics of Relativity, Volume 84 of Philosophical Studies Series. Springer, Dordrecht (2001)

7. Craig, W.L., Smith, Q.: Einstein Relativity and Absolute Simultaneity. Routledge, New York (2008)

8. Dieks, D.: Time, space, spacetime. Metascience 21(3), 617-619 (2012). https://doi.org/10.1007/ s11016-012-9690-1

9. Dieks, D.: Time in special relativity. In: Ashtekar, A., Petkov, V. (eds.) Springer Handbook of Spacetime. Book Section 6, pp. 91-113. Springer, Berlin (2014)

10. Dürr, D., Goldstein, S., Norsen, T., Struyve, W., Zanghì, N.: Can bohmian mechanics be made relativistic? Proc. R. Soc. A 470, 2162 (2014). https://doi.org/10.1098/rspa.2013.0699

11. Einstein, A.: Relativity: The Special and the General Theory—A Popular Exposition. Methuen, London (1920)

12. Einstein, A.: On the electrodynamics of moving bodies. In: Stachel, J., Cassidy, D.C., Renn, J., Schulmann, R. (eds.) The Collected Papers of Albert Einstein-English Translations, vol. 2, pp. 140-171. Princeton University Press, Princeton (1989)

13. Gilmore, C., Costa, D., Calosi, C.: Relativity and three four-dimensionalisms. Philos. Compass 11(2), 102 (2016)

14. Godfrey-Smith, W.: Special relativity and the present. Philos. Stud.: Int. J. Philos. Anal. Trad., 36(3):233-244 (1979). ISSN 00318116, 15730883. http://www.jstor.org/stable/4319321. Accessed 29 Aug 2019

15. Grünbaum, A.: Logical and philosophical foundations of the special theory of relativity. Am. J. Phys. 23, 450-464 (1955)

16. Harrington, J.: Special relativity and the future: a defense of the point present. Stud. Hist. Philos. Sci. B, 39(1):82-101 (2008). ISSN 1355-2198. https://doi.org/10.1016/j.shpsb.2007.06.003. http://www. sciencedirect.com/science/article/pii/S1355219807000627. Accessed 29 Aug 2019 
17. Hinchliff, M.: The puzzle of change. Philos. Persp., 10:119-136 (1996). ISSN 15208583, 17582245. https://doi.org/10.2307/2216239. http://www.jstor.org/stable/2216239. Accessed 29 Aug 2019

18. Hinchliff, M.: A defense of presentism in a relativistic setting. Philosophy of Science 67, S575-S586 (2000). https://doi.org/10.1086/392847. http://www.journals.uchicago.edu/doi/abs/10.1086/392847. Accessed 29 Aug 2019

19. Jammer, M.: Concepts of Simultaneity: From Antiquity to Einstein and Beyond. Johns Hopkins University Press, Baltimore (2006)

20. Malament, D.: Causal theories of time and the conventionality of simultaneity. Nous 11, 293-300 (1977)

21. Monton, B.: Presentism and quantum gravity. In: Dieks, D. (ed.) The Ontology of Spacetime. Book Section 14, pp. 263-280. Elsevier, Amsterdam (2006)

22. Norton, J.D.: Philosophy of space and time. In: Salmon, M.H., Earman, J., Glymour, C., Lennox, J.G., Machamer, P., McGuire, J.E., Norton, J.D., Salmon, W.C., Schaffner, K.F. (eds.) Introduction to the Philosophy of Science, Book Section 5. Hackett Publishing Company, Indianapolis (1992)

23. Norton, J.D.: Einstein for Everyone. Nullarbor Press, Pittsburgh (2018)

24. Penrose, R.: The Emperor's New Mind. Oxford University Press, Oxford (1989)

25. Peterson, D., Silberstein, M.: Relativity of simultaneity and eternalism: in defense of the block universe. In: Petkov, V. (ed.) Space. Time, and Spacetime, vol. 167, pp. 209-237. Springer-Verlag, Berlin (2010)

26. Petkov, V.: Simultaneity, conventionality and existence. Br. J. Philos. Sci., 40(1):69-76 (1989). ISSN 00070882, 14643537. http://www.jstor.org/stable/687464. Accessed 29 Aug 2019

27. Petkov, V.: Conventionality of simultaneity and reality. In: Dieks, D. (ed.) The Ontology of Spacetime II. Book Section 9, pp. 175-185. Elsevier, Amsterdam (2008)

28. Price, H.: Time's Arrow and Archimedes' Point. Oxford University Press, Oxford (1996)

29. Putnam, H.: Time and physical geometry. J. Philos. 64(8), 240-247 (1967)

30. Redhead, M.: The conventionality of simultaneity. In: Earman, J., Janis, A.I., Massey, G.J., Rescher, N. (eds.) Philosophical Problems in the Internal and External World: Essays in the Philosophy of Adolf Grünbaum, pp. 103-128. University of Pittsburgh Press, Pittsburgh (1993)

31. Reichenbach, H.: Der gegenwärtige stand der relativitätsdiskussion. Logos 10, 316-378 (1922)

32. Reichenbach, H.: Axiomatik der relativistischen Raum-Zeit-Lehre. Vieweg, Braunschweig (1924)

33. Reichenbach, H.: Philosophie der Raum-Zeit-Lehre. W. de Gruyter, Berlin (1928)

34. Reichenbach, H.: The Philosophy of Space and Time. Dover Publications, New York (1958)

35. Reichenbach, H.: On the present state of the discussion on relativity. In: Reichenbach, M. (eds), Modern Philosophy of Science-Selected Essays, vol. 2, pp. 3-47. Routledge and Kegan Paul, London (1959)

36. Reichenbach, H.: Axiomatization of the Theory of Relativity. Univeristy of California Press, Berkeley (1969)

37. Rietdijk, C. W.: A rigorous proof of determinism derived from the special theory of relativity. Philos. Sci., 33(4):341-344 (1966). ISSN 00318248, 1539767X. http://www.jstor.org/stable/186637. Accessed 29 Aug 2019

38. Saunders, S.: How relativity contradicts presentism. R. Inst. Philos. Suppl. 50, 277 (2002)

39. Savitt, S.: There's no time like the present (in Minkowski spacetime). Philos. Sci., 67:S563-S574, (2000). ISSN 00318248, 1539767X. http://www.jstor.org/stable/188695. Accessed 29 Aug 2019

40. Savitt, S.: Being and Becoming in Modern Physics. In: Edward, N.Z. (ed.) The Stanford Encyclopedia of Philosophy. Stanford University, Metaphysics Research Lab (2014). https://plato.stanford.edu/ archives/fall2017/entries/spacetime-bebecome/. Accessed 29 Aug 2019

41. Sklar, L.: Space, Time, and Spacetime. University of California Press, Berkeley (1974)

42. Sklar, L.: Time, reality and relativity. In: Healy, R. (ed.) Reduction. Time and Reality, pp. 129-142. Cambridge University Press, New York (1981)

43. Sklar, L.: Philosophy and Spacetime Physics. University of California Press, Berkeley (1985)

44. Stein, H.: On Einstein-Minkowski space-time. J. Philos. 67, 5-23 (1968)

45. Torretti, R.: Relativity and Geometry. Foundations and Philosophy of Science and Technology Series. Pergamon Press, Oxford (1983)

46. Weingard, R.: Relativity and the reality of past and future events. Br. J. Philos. Sci. 23(2), 119-121 (1972). https://doi.org/10.1093/bjps/23.2.119

Publisher's Note Springer Nature remains neutral with regard to jurisdictional claims in published maps and institutional affiliations. 